\title{
Aldosterone Induces Apoptosis in Rat Podocytes: Role of PI3-K/Akt and p38MAPK Signaling Pathways
}

\author{
Cheng Chen ${ }^{a}$ b Wei Liang ${ }^{a}$ Junya Jia $^{a}$ Harry van Goor ${ }^{b}$ Pravin C. Singhal ${ }^{c}$ \\ Guohua Ding ${ }^{a}$ \\ ${ }^{a}$ Division of Nephrology, Renmin Hospital of Wuhan University, Wuhan, China; ${ }^{b}$ Department of Pathology, \\ University Medical Center Groningen, University of Groningen, Groningen, The Netherlands; \\ 'Department of Medicine, Long Island Jewish Medical Center, New Hyde Park, N.Y., USA
}

\section{Key Words}

Aldosterone $\cdot$ Podocyte $\cdot$ Phosphatidylinositol 3-kinase/Akt • p38 mitogen-activated protein kinase $\cdot$ Apoptosis

\begin{abstract}
Background: Podocytes play a critical role in the pathogenesis of glomerulosclerosis. Increasing evidence suggests that aldosterone (ALD) is involved in the initiation and progression of glomerular damage. It is, however, unknown whether there is a direct injurious effect of ALD on podocytes. Therefore, in the present study, we evaluated the effect of ALD on podocyte apoptosis and studied the role of phosphatidylinositol 3-kinase/Akt (PI3-K/Akt) and p38 mitogen-activated protein kinase (p38MAPK) signaling pathways in this process. Methods: Podocytes were incubated in media containing either buffer or increasing concentrations of ALD $\left(10^{-9} \sim 10^{-5} \mathrm{M}\right)$ for variable time periods. The cells were also treated with either wortmannin (inhibitor of PI3-K, 100 nM), SB202190 (SB20, inhibitor of P38MAPK, $10 \mu \mathrm{M}$ ) or buffer. All treatments were performed with or without ALD $\left(10^{-7} \mathrm{M}\right)$ for $24 \mathrm{~h}$. At the end of the incubation period, apoptosis was evaluated by cell nucleus staining and flow cytometric analyses. Activation of PI3-K/Akt and p38MAPK phosphorylation of cultured rat podocytes was evaluated by performing Akt
\end{abstract}

kinase assay and Western blot, respectively. Results: Apoptosis of cultured rat podocytes was induced by ALD in a dose- and time-dependent manner. ALD inhibited the activity of PI3-K/Akt and increased the activation of p38MAPK. PI3-K/Akt activity was further inhibited by the addition of wortmannin to the cells in the presence of ALD. This was accompanied by a significant increase in apoptosis. ALD-induced p38MAPK phosphorylation and apoptosis were inhibited when the cells were pretreated with SB20. Furthermore, treatment with spironolactone not only attenuated the proapoptotic effect of ALD, but also significantly reversed its effects on PI3-K/Akt and p38MAPK signaling pathways. Conclusion: ALD induces apoptosis in rat podocytes through inhibition of PI3-K/Akt and stimulation of p38 MAPK signaling pathways. Spironolactone attenuates ALD-induced podocyte apoptosis, thereby positioning this compound as a potential promising target of intervention in human renal damage.

Copyright $\odot 2009$ S. Karger AG, Basel

C.C. and W.L. contributed equally to this study. This work was presented in part at the 34th Annual Meeting of the American Society of Nephrology, 2006, San Diego, Calif., USA.

\section{KARGER}

(C) 2009 S. Karger AG, Basel

Fax +41613061234

E-Mail karger@karger.ch

www.karger.com
Accessible online at: www.karger.com/nee
Guohua Ding, MD, PhD

Division of Nephrology, Renmin Hospital of Wuhan University

238 Jiefang Road

Wuhan 430060 (China)

Tel. +86 278804 1911, Fax +86 278804 2292, E-Mail gxd101@yahoo.com 


\section{Introduction}

Aldosterone (ALD) is an important mediator of the renin-angiotensin-ALD system (RAAS) and plays a pivotal role in the regulation of salt and extracelluar fluid metabolism [1]. ALD also has nonhemodynamic effects leading to end organ damage and renal injury [2]. These detrimental effects of ALD are independent of the RAAS. Rats with hypertension, diabetic nephropathy or glomerulosclerosis exhibit high levels of ALD associated with proteinuria and progressive renal injury $[3,4]$. ALD induces production of reactive oxygen species and inflammatory molecules in hypertensive rats [5]. It also stimulates excretion of TGF- $\beta$ into the urine [6]. Moreover, inhibitors of ALD or adrenalectomy attenuate renal injury in human nephropathies as well as various experimental animal models $[7,8]$.

Plasma and tissue ALD are elevated in diabetic and other progressive nephropathies and may cause various physiological effects through both genomic and nongenomic mechanisms [9]. Several studies have suggested that the mineralocorticoid receptor (MR) and the membrane receptor may be involved. Genomic action is mainly through the type $1 \mathrm{MR}$ which locates on the nuclear membrane. The ALD-MR complex is the classical ALD pathway leading to inhibition or activation of several target genes, whereas the nongenomic actions of ALD are mediated by binding cellular membrane sites [10]. Those effects are more rapid and stronger than the genomic ones while the membrane receptor has not been definitely identified and the mechanisms leading to those effects remain not well defined.

Podocytes are highly differentiated cells and constitute the main component of the glomerular filtration barrier. Its injury, apoptosis or depletion from glomerular basement membrane are thought to be associated with the initiation and progression of glomerulosclerosis [11]. Several studies demonstrated that a variety of pathophysiological stimuli such as TGF- $\beta$ [12], angiotensin II [13], intoxicant [14] and reactive oxygen species [15] can induce podocyte apoptosis in cultured cells and experimental animal models. Apoptosis induced by ALD has been reported in vivo and in vitro in a variety of cells including endothelial cells and myocytes $[16,17]$, but not in podocytes.

Since podocyte apoptosis is crucial in the development of renal fibrosis, we investigated the potential role of ALD in the induction of podocyte apoptosis in cultured rat podocytes. Furthermore, we studied the effects of ALD on the main anti- or proapoptotic signaling pathways, i.e. phosphatidylinositol 3-kinase/Akt (PI3-K/Akt) and p38 mitogen-activated protein kinase (p38MAPK) in ALDstimulated podocyte apoptosis.

\section{Methods}

\section{Podocyte Culture}

Primary cultures of podocytes were prepared from isolated glomeruli of Sprague-Dawley rats (Experimental Animal Center of Wuhan University) according to a previously described technique with slight modifications [18]. Briefly, glomeruli were isolated by sieving of minced cortices. Glomeruli were plated and cultured on type I collagen-coated culture flasks (BD Falcon, Bedford, Mass., USA) in media consisting of equal parts of conditioned 3T3 media and Dulbecco's modified Eagle's medium nutrient mixture F-12 HAM (Gibco, Carlsbad, Calif., USA), supplemented with insulin-transferrin-selenium media supplement (Gibco), 5\% fetal bovine serum (Sigma, St. Louis, Mo., USA), penicillin $(100 \mathrm{U} / \mathrm{ml})$ and streptomycin $(100 \mu \mathrm{g} / \mathrm{ml})$ at $37^{\circ} \mathrm{C}$ in $5 \%$ $\mathrm{CO}_{2}$. After 5 days, cell outgrows from the attached glomeruli were digested, passed through a sieve with a pore size of $40 \mu \mathrm{m}$ and transferred to another flask. Podocytes were characterized by immunofluorescent positive staining for podocalyxin, synaptopodin and WT1 (Santa Cruz Biotechnology, Santa Cruz, Calif., USA), in combination with negative staining for myosin and factor VIII. Podocytes were used for experiments between passages 3 and 6 .

The conditionally immortalized mouse podocyte cell line (MPC5) was kindly provided by Dr. Peter Mundel (Mount Sinai School of Medicine, New York, N.Y., USA). To induce proliferation, podocytes were cultured at $33^{\circ} \mathrm{C}$ in RPMI 1640 medium, supplemented with $10 \%$ fetal bovine serum and $10 \mathrm{U} / \mathrm{ml}$ mouse recombinant interferon- $\gamma$ (ImmunoKontact, Abingdon, UK) to boost the expression of thermosensitive large-T antigen. To induce differentiation, podocytes were seeded on collagen type I coated plates without interferon- $\gamma$ under nonpermissive conditions at $37^{\circ} \mathrm{C}$ for 10 days.

\section{Experimental Treatment of Podocytes}

After the characterization, equal numbers of cells were plated and grown to subconfluence. Podocytes were washed twice with PBS and then incubated in media (1\% FCS) containing buffer (control), variable concentrations of ALD $\left(10^{-9}\right.$ to $10^{-5} \mathrm{M}$; Sigma), or stable concentration of ALD $\left(10^{-7} \mathrm{M}\right.$; Sigma) for 6, 12, 18, or $24 \mathrm{~h}$. In separate experiments, podocytes were pretreated with wortmannin $(100 \mathrm{nM}$, a specific antagonist of p85, regulatory subunit of PI3-K; Cell Signaling Technology, Danvers, Mass., USA), SB20 (10 $\mu \mathrm{M}$, a selective p38MAPK antagonist; EMD Bioscience, Darmstadt, Germany), or spironolactone (SPIR; $10^{-7} \mathrm{M}$, a blocker of ALD; Sigma) for $1 \mathrm{~h}$ prior to the stimulation with ALD $\left(10^{-7} \mathrm{M}\right)$.

\section{Flow Cytometry by Annexin V-FITC Conjugated with PI \\ Staining}

Podocyte apoptosis was determined by flow cytometry, using annexin V-FITC and propidium iodide (PI) double staining [19]. As recommended by the manufacturer of annexin V-FITC apoptosis detection kit I (BD Biosciences, San Jose, Calif., USA), cells in each treated group were washed twice with cold PBS and then 
resuspended in binding buffer. A total of $1 \times 10^{6}$ cells $/ \mathrm{ml}$ were transferred in a 5 -ml tube containing $5 \mu l$ of annexin V-FITC and $5 \mu \mathrm{l}$ of PI and were incubated for $15 \mathrm{~min}$ at room temperature in the dark. After adding $400 \mu \mathrm{l}$ of binding buffer, the samples were analyzed by flow cytometry (BD FACSCalibur, Rockville, Md., USA) within $1 \mathrm{~h}$. Annexin V-positive and PI-negative cells were defined as apoptotic. A total of $2 \times 10^{4}$ cells were detected by FCM in each of the samples. Data analysis was performed with Modifit Flow Cytometry Software.

\section{Detection of Apoptosis by H-33342 Staining}

Morphological evaluation of podocyte apoptosis was performed using H-33342 (Sigma) staining which detects the nuclei of both apoptotic and living cells. Apoptotic cells in treated groups exhibited nuclear condensation, nuclear fragmentation and chromatin margination. The condensed nuclei were stained bright blue with increased fluorescence. After incubation with the various components as outlined in 'Experimental Treatment of Podocytes', cells were fixed with $95 \%$ alcohol for $15 \mathrm{~min}$ and stained with $\mathrm{H}-33342(10 \mu \mathrm{g} / \mathrm{ml})$ for $5 \mathrm{~min}$ at $37^{\circ} \mathrm{C}$ in the dark. The stained cells were studied using a laser confocal microscope (Leica, Wetzlar, Germany). The rate of apoptotic cells was recorded in ten random nonoverlapping fields by two blinded observers.

\section{Protein Extraction and Western Blot Analysis}

Western blot analysis was carried out as described previously [20]. In brief, primary cultured podocytes were lysed on ice in cell lysis buffer (150 mM NaCl, 1 mM EDTA, 1 mM EGTA, 1\% Triton$100,2.5 \mathrm{mM}$ sodium pyrophosphate, $1 \mathrm{mM} \mathrm{Na}_{3} \mathrm{VO}_{4}, 1 \mathrm{mM} \beta$-glycerophosphate, $1 \mu \mathrm{g} / \mathrm{ml}$ leupeptin, $1 \mathrm{mM}$ phenylmethyl sulfonylfluoride, $20 \mathrm{~mm}$ Tris- $\mathrm{HCl}$, pH 7.5) for $30 \mathrm{~min}$. The cell lysates were centrifuged at $14,000 \mathrm{~g}$ for $20 \mathrm{~min}$ in a $4^{\circ} \mathrm{C}$ centrifuge. The protein concentration was determined using a BCA protein assay kit (Pierce, Rockford, Ill., USA). Equal amounts of proteins $(25 \mu \mathrm{g})$ were subjected to a $12 \%$ SDS-polyacrylamide gel. After being electrophoresed, the proteins were transferred onto a PVDF membrane by using a Bio-Rad Western blot analysis apparatus. The membrane was incubated with blocking buffer for $60 \mathrm{~min}$ at room temperature and then incubated overnight at $4^{\circ} \mathrm{C}$ with the primary polyclonal antibodies against p38, phospho-p38 MAPK (New England Biolabs, Herts, UK) with $\beta$-actin as a normalized reference, followed by the addition of horseradish peroxidase (HRP)-labeled secondary antibodies. The peroxidase activity was visualized by using a chemiluminescence detection kit (ECL; Santa Cruz) and exposed to Kodak film. Quantitative densitometry was performed by using a computer-based image analysis system.

\section{Akt/Protein Kinase B Kinase Activity}

Akt/protein kinase B (PKB) kinase activity was measured by Akt/PKB kinase assay kit (Cell Signaling Technology). Immobilized Akt is used to immunoprecipitate Akt from cell extracts. Then, an in vitro kinase assay is performed using GSK-3 fusion protein as a substrate. The Akt kinase activity was evaluated by the phosphorylation level of GSK-3 $\alpha / \beta$ (Ser21/9). In brief, based on different experimental conditions cells were lysed in the same way as protein extraction above. Immunoprecipitation was performed by using $20 \mu \mathrm{l}$ immobilized anti-Akt IgG1 monoclonal antibody incubated with $200 \mu \mathrm{l}$ cell lysate overnight at $4^{\circ} \mathrm{C}$. After microcentrifugation and washing the cell lysis buffer and kinase buffer, respectively, $1 \mu \mathrm{l}$ of $10 \mathrm{mM}$ ATP and $1 \mu \mathrm{g}$ of GSK-3 fusion protein were mixed with the pellet in $50 \mu \mathrm{l}$ kinase buffer, incubated for $30 \mathrm{~min}$ at $30^{\circ} \mathrm{C}$. The reaction was ended and the mixture was microcentrifuged for Western blot analysis. Phospho-GSK-3a/b rabbit polyclonal antibody (Ser21/9, dilution 1:1,000) was used as a primary antibody and HRP-conjugated anti-rabbit IgG was used as secondary antibody (dilution $1: 2,000)$.

\section{Statistical Analysis}

Results are presented as means \pm SEM. Statistical comparisons were made using one-way ANOVA followed by Student's $t$ test or Dunnett's test using SPSS v11.0 software. Statistical significance was defined as a $\mathrm{p}$ value $<0.05$.

\section{Results}

\section{ALD Induces Apoptosis in Primary Cultured Podocytes and MPC5 Cells}

As shown in figure 1 and $2 \mathrm{a}$, ALD induced apoptosis in primary cultured podocy tes in a dose-dependent manner. In figure 2a, apoptosis of MPC5 cells treated with $10^{-5} \mathrm{M}$ ALD was less than when treated with $10^{-7} \mathrm{M}$. This might be caused by the direct induction of necrosis by this high dose of ALD. During the time course effect of $\operatorname{ALD}\left(10^{-7} \mathrm{M}\right)$ on primary cultured podocytes and MPC5 cell apoptosis, ALD induced apoptosis of primary cultured podocytes and MPC4 in a time-dependent manner. As shown in figures 1 and $2 \mathrm{~b}, \operatorname{ALD}\left(10^{-7} \mathrm{M}\right)$ promoted primary cultured podocytes and MPC5 cell apoptosis in a time-dependent manner. Apoptosis was quantified for each treatment condition in triplicate. Hoechst-33342 staining displayed the morphologic changes of podocyte nuclei under the laser confocal microscope (fig. 3). Figure 3 shows the presence of ALD-induced podocyte apoptosis (1.81 vs. $24.44 \%$ ).

\section{ALD Suppresses Activation of PI3-K/Akt}

The activation of Akt is reflected by testing the phosphorylation level of GSK-3 $\alpha / \beta$ after Akt immunocoprecipitation has been performed. Western blot analysis showed (fig. 4a) that incubation of podocytes with ALD significantly inhibited the activation of Akt in a dose-dependent manner, with the minimum activation at approximately $60 \mathrm{~min}$, and returning to almost normal levels at $120 \mathrm{~min}$ (fig. 4b). We therefore investigated the effect of podocyte pretreatment with wortmannin on Akt activation and apoptosis. Akt was inactivated and associated with increased podocyte apoptosis when this specific inhibitor of PI3-K was added (fig. 4c). Incubation with SB20 caused no significantly change in the activa- 


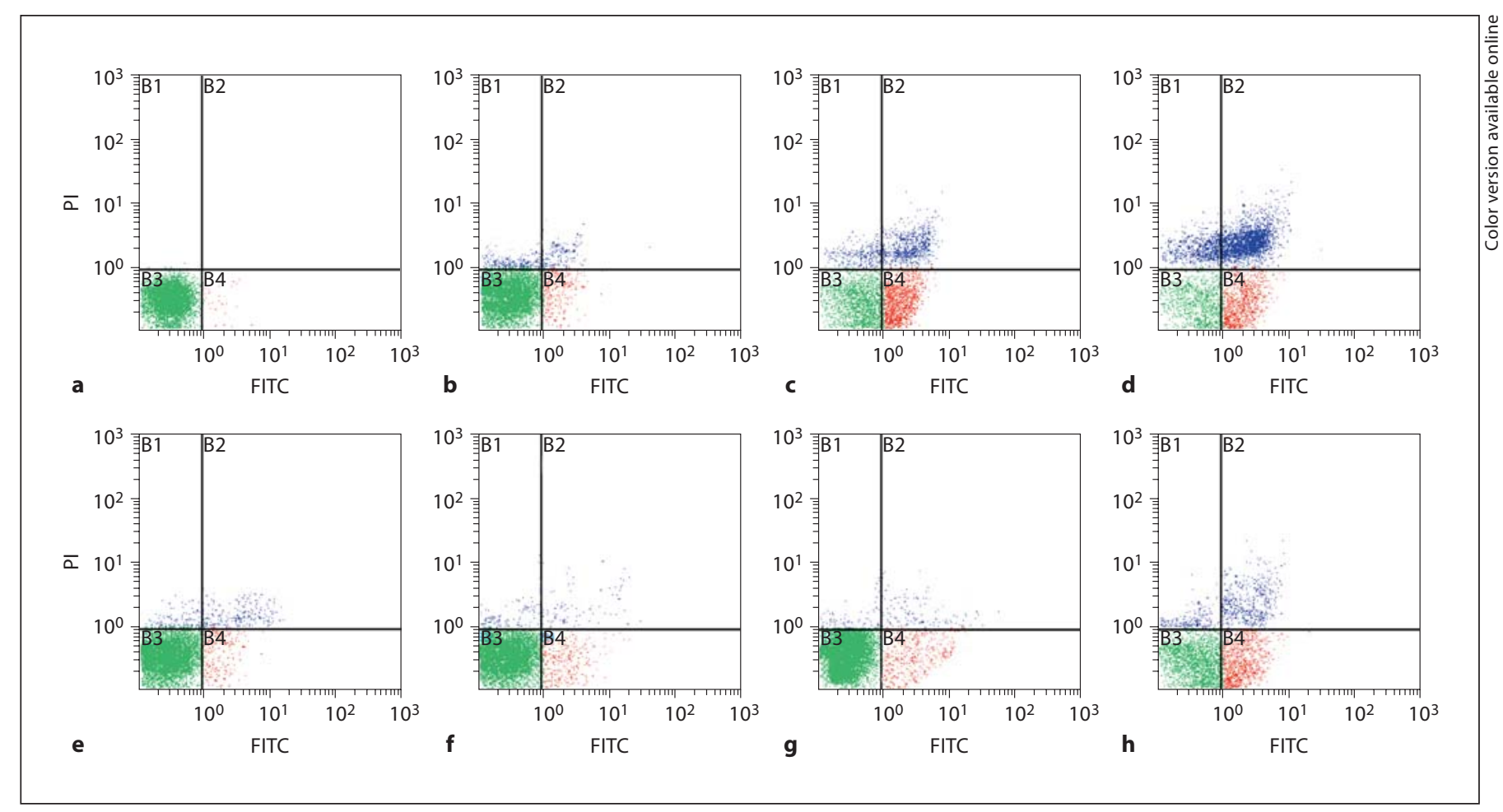

Fig. 1. Dose- and time-dependent apoptosis induced by ALD in podocytes. a-d Dose-response effect of ALD on podocyte apoptosis. Equal numbers of subconfluent podocytes were incubated in media containing either buffer (as a control; a) or variable concentrations of $\operatorname{ALD}\left(10^{-9}\right.$ to $\left.10^{-5} \mathrm{M}, \mathbf{b}-\mathbf{d}\right)$ for $24 \mathrm{~h}$. At the end of the incubation period, the extent of apoptosis was determined by flow cytometry using PI for hypodiploid DNA and annexin V double staining. $\mathrm{X}$-axis represents FITC staining and $\mathrm{y}$-axis represents $\mathrm{PI}$ staining. Left lower quadrant means annexin V-FITC and PI negative. Right lower quadrant means annexin-V FITC positive and
PI negative. Right superior quadrant means annexin V-FITC and PI positive. Left superior quadrant means PI positive. Viable cells were annexin V-FITC and PI negative, cells in early-stage apoptosis were annexin-V FITC positive and PI negative, cells in latestage apoptosis and already dead were annexin V-FITC and PI positive. e-h Time response effects of ALD on podocyte apoptosis. Equal numbers of subconfluent podocytes were treated with $\operatorname{ALD}\left(10^{-7} \mathrm{M}\right)$ for $6(\mathbf{e}), 12(\mathbf{f}), 18(\mathbf{g})$ and $24 \mathrm{~h}(\mathbf{h})$. At the confining point, cells were stained as described above. tion of Akt. SPIR treated with podocytes prevented ALDinduced Akt inactivation.

\section{ALD Triggers the Activation of $p 38 M A P K$}

As shown in figure $5 \mathrm{a}$ and $\mathrm{b}$, ALD increased the activation of p38MAPK in a time- and concentration-dependent manner. We further evaluated whether the two pathways (p38MAPK and A13-K/Akt) were associated with each other. The specific inhibitor of p38MAPK, SB20, was used to assess the effect of p38MAPK on PI3K/Akt. Incubation of the cells with SB20 partially abolished the proapoptotic effect of ALD (fig. 5c), but it had no influence on Akt activation, verifying that ALD induced podocyte apoptosis through p38MAPK pathway and PI3-K/Akt pathway independently. SPIR prevented p-p38 formation during ALD stimulation (fig. 5c).

\section{Protective Effect of SPIR}

As SPIR could affect several genes associated with cell survival, flow cytometry followed by annexin V-FITC and PI double staining were carried out to investigate whether SPIR affects ALD-induced podocyte apoptosis. SPIR- and ALD-treated podocytes revealed less apoptosis compared with podocytes treated with ALD alone. This was further evidenced by morphological analysis (fig. 6).

\section{Discussion}

Based on their special cytobiological characteristics, podocytes play a pivotal role in maintaining the integrality of the glomerular filtration barrier [21]. Numerous 


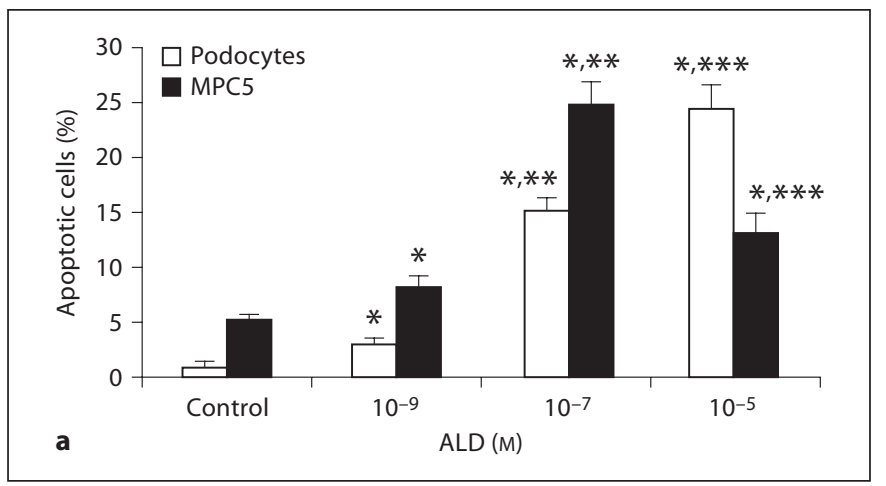

Fig. 2. Dose-response (a) and time course (b) effect of ALD on primary cultured podocyte and MPC5 cell apoptosis. a Equal numbers of cells were incubated in media containing either buffer (control) or $10^{-9}$ and $10^{-5} \mathrm{M}$ ALD for $24 \mathrm{~h}$. At the end of the incubation period, cells were stained for apoptosis. The concentration of $10^{-5} \mathrm{M}$ ALD was so high to MPC5 cells that it caused necrosis directly. Values are mean \pm SE of 3 sets of experiments, each car-

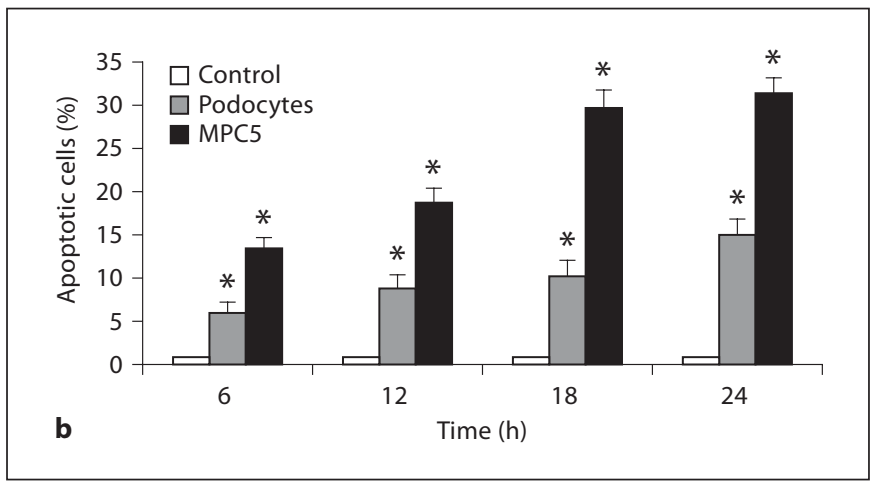

ried out in triplicate. ${ }^{*} \mathrm{p}<0.05$ vs. control; ${ }^{* *} \mathrm{p}<0.05$ vs. $10^{-9} \mathrm{M}$ ALD; ${ }^{* * *} \mathrm{p}<0.05$ vs. $10^{-7}$ M ALD. b Time course effect of ALD $\left(10^{-7} \mathrm{M}\right)$ on primary cultured podocyte and MPC5 cell apoptosis. Equal numbers of cells were incubated in media containing either buffer (control) or $10^{-7} \mathrm{M}$ ALD for variable time periods $(6,12,18$, and $24 \mathrm{~h}$ ). At the end of the incubation period, cells were stained for apoptosis. ${ }^{*} \mathrm{p}<0.05$, compared with respective controls.
Fig. 3. Hoechst-33324 staining of podocytes. Representative fluorescence microscopy of Hoechst-33342 staining at $24 \mathrm{~h}$ after incubation of podocytes in media containing either buffer (as control; a) or variable concentrations of ALD $\left(10^{-9}, 10^{-7}\right.$, $10^{-5} \mathrm{M} ; \mathbf{b}-\mathbf{d}$, respectively). a In the control group, $1.81 \%$ of podocytes underwent apoptosis. b-d ALD increased podocyte apoptosis at most to $24.44 \%$ in ten random nonoverlapping fields. b Bright and rippled nuclei were observed, indicating the nature of early stage of apoptosis. c, d High condensation and marginalization became increasingly clear in the nuclei, indicating the nature of late stage of apoptosis.
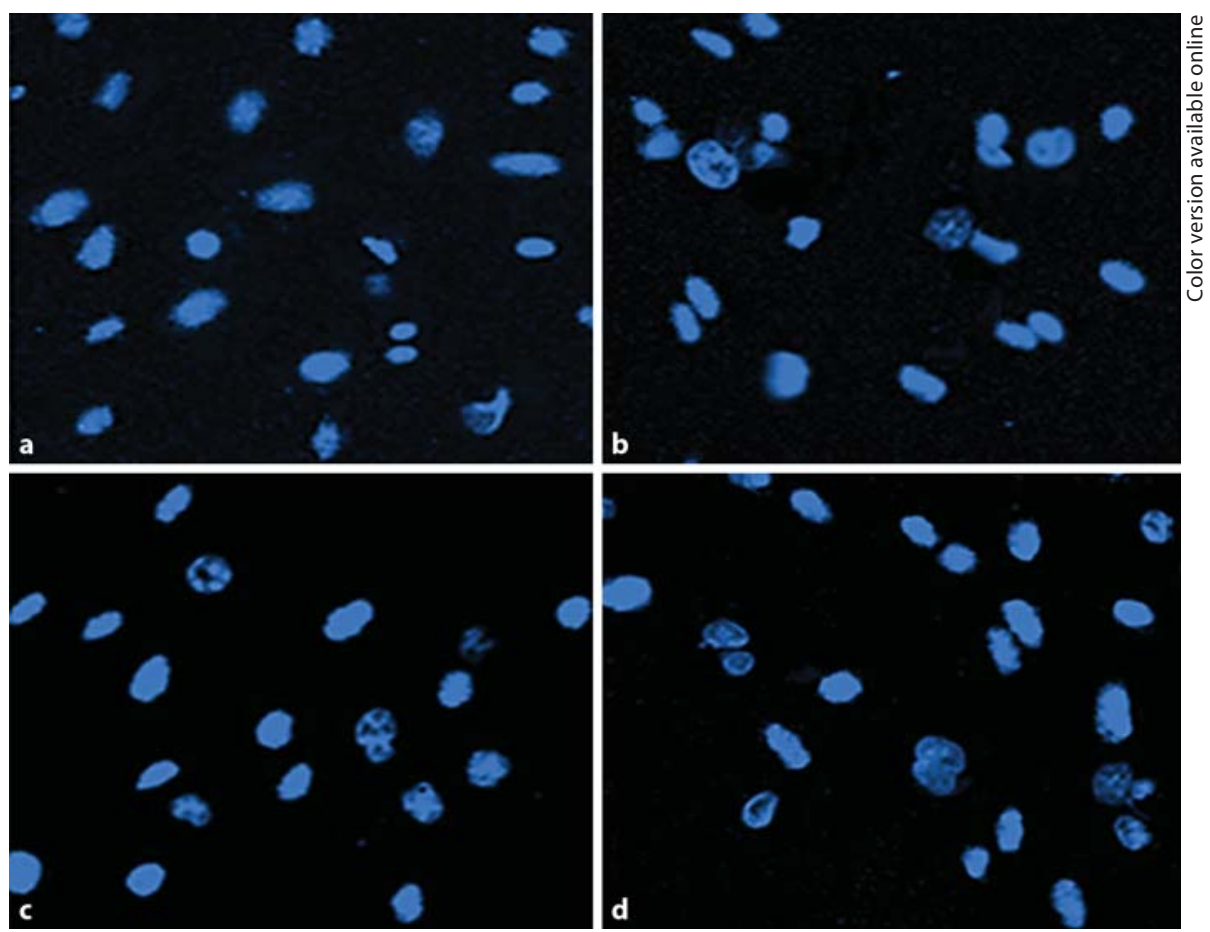

studies have reported that various deleterious factors such as viral infection, oxidative damage and mechanical stress can induce podocyte injury. However, the consequences of ALD exposure to podocytes and the mechanisms by which ALD contributes to pathological processes are poorly understood. The present study demonstrates that ALD can directly induce primary cultured podocyte apoptosis in a dose- and time-dependent manner and has a similar effect on MPC5 cells. The process was induced via activation of $\mathrm{p} 38$ MAPK and inhibition of PI3-k/Akt pathways. Furthermore, ALD-induced apoptosis was prevented by coincubation with SB20, an inhibitor of 


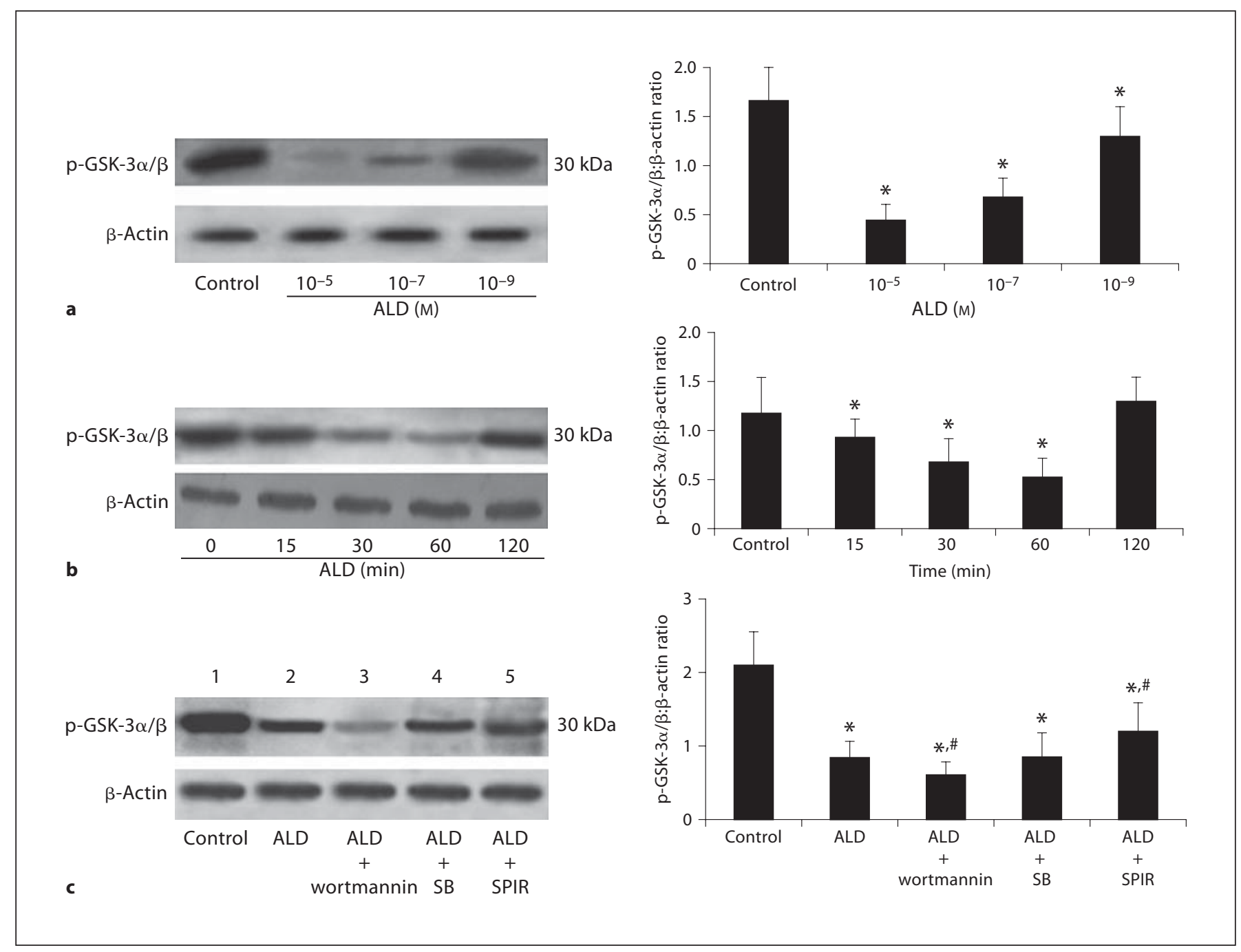

Fig. 4. Western blot assay for Akt in podocytes. a ALD decreases activation of $\mathrm{p}-\mathrm{GSK}-3 \alpha / \beta$ which can reflect the activation of Akt. Podocytes were treated with either buffer (as control) or variable concentrations of ALD $\left(10^{-5}\right.$ to $\left.10^{-9} \mathrm{M}\right)$. Cell lysates were prepared and used for measuring phosphorylated levels of GSK-3 $\alpha / \beta$,or $\beta$ actin immunoblot using respective antibodies. $\mathbf{b}$ Effects of ALD on Akt expression in podocytes. Podocytes were treated with or without $\operatorname{ALD}\left(10^{-7} \mathrm{M}\right)$ for the indicated times ( 0 as control). At each time point, whole cell lysates were prepared and used for $\mathrm{p}$ -
GSK- $3 \alpha / \beta$ or $\beta$-actin immunoblot using respective antibodies. c Effects of wortmannin, SB202190 and SPIR on ALD-induced Akt expression in podocytes. Podocytes were pretreated for $1 \mathrm{~h}$ with wortmannin (lane 3), SB202190 (lane 4), or SPIR (lane 5) before being treated with buffer (as control, lane 1) or ALD $\left(10^{-7} \mathrm{M}\right.$, lanes 2-5) for $60 \mathrm{~min}$. Whole cell lysates were prepared and analyzed by immunoblot to detect $\mathrm{p}-\mathrm{GSK}-3 \alpha / \beta$. ${ }^{*} \mathrm{p}<0.05 \mathrm{vs}$. control group; ${ }^{\#} \mathrm{p}<0.05$ vs. ALD group.
p38MAPK and aggravated by wortmannin, the p85 subunit of PI3-K inhibitor.

In a previous study, we found that angiotensin II also induces podocyte apoptosis [13]. Recent studies have shown that ALD, besides its classic effect as a downstream factor of angiotensin II in the distal collecting duct of the kidney on sodium retention and potassium loss, can also stimulate a number of signaling cascades with both phys- iologic and pathophysiologic consequences. ALD can increase inflammatory mediators, oxidant stress [22] and negatively affect endothelial function in the cardiovascular system. These effects are not only caused by circulating ALD synthesized by the adrenal gland, but also by local biosynthesis as occurs in blood vessels [8]. In the heart, it contributes to the pathophysiology of heart failure by initiating cardiomyocyte apoptosis [16]. Podocytes 


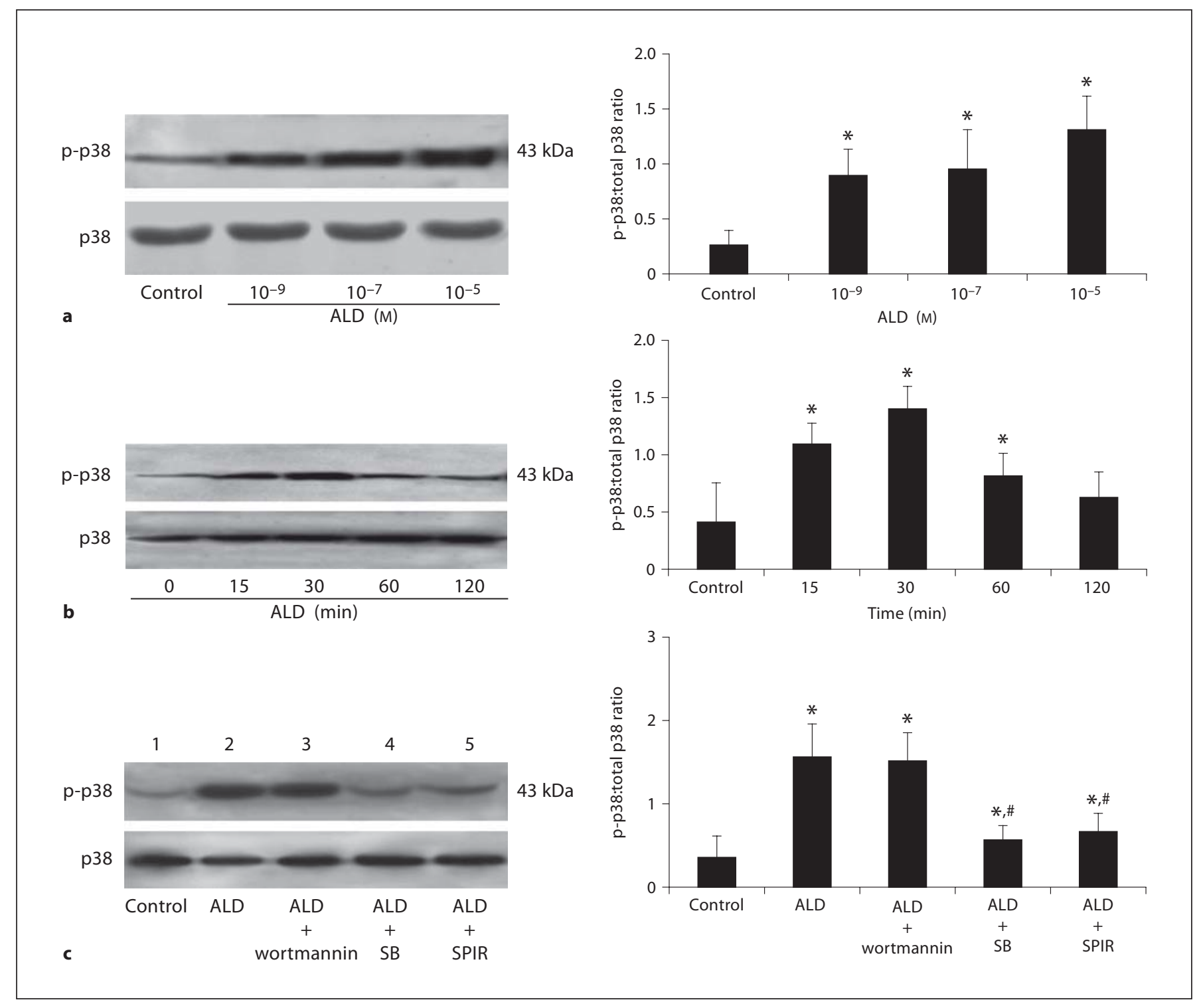

Fig. 5. Western blot assay for phosphorylated $\mathrm{p} 38 \mathrm{MAPK}$ in podocytes. Podocytes were treated with either $10^{-9}, 10^{-7}$, or $10^{-5} \mathrm{M}$ ALD (a) or $10^{-7} \mathrm{M} \mathrm{ALD} \mathrm{(b)} \mathrm{for} \mathrm{0,} \mathrm{15,} \mathrm{30,} \mathrm{60,} 120 \mathrm{~min}$. Cell lysates were prepared and immunoblotted to measure phosphorylated and total levels of p38MAPK as indicated. c Effects of wortmannin, SB202190 and SPIR on ALD-induced p38MAPK expression in

are very fragile and injury could occur through various deleterious stimuli, resulting in podocyte hiatus and apoptosis, which subsequently play a vital role in the development of glomerulosclerosis [13, 23]. In renal failure and diabetic nephropathy models, ALD is associated with albuminuria [3], hypertension [24] and glomerulosclerosis [7] as an independent agent. Nagase et al. [25] and Nagase and Fujita [26] reported recently that ALD podocytes. Podocytes were pretreated for $1 \mathrm{~h}$ with wortmannin (lane 3), SB202190 (lane 4), or SPIR (lane 5) before being treated with buffer (as control, lane 1$)$ or $\operatorname{ALD}\left(10^{-7} \mathrm{M}\right.$, lanes $\left.2-5\right)$ for 30 $\mathrm{min}$. Whole cell lysates were prepared and analyzed by immunoblot to detect p-p38MAPK and total p38MAPK. ${ }^{*}$ p $<0.05$ vs. control group; ${ }^{\#} \mathrm{p}<0.05$ vs. ALD group.

evoked glomerular podocyte injury through oxidative stress and sgk1 upregulation.

PKB (also referred to as Akt or Rac) [27] is the major component of the serine-threonine protein kinase family which plays an important role in apoptosis and survival signaling pathways [28]. Akt is preferentially activated in a PI3-K-dependent manner by phosphorylation at Thr308 and Ser473. This activation plays a pivotal role 
Fig. 6. Effect of wortmannin, SB202190 and SPIR on ALD-induced podocyte apoptosis. Equal numbers of cells were pretreated for $1 \mathrm{~h}$ with wortmannin, SB202190 and SPIR, respectively, before treatment with or without $\operatorname{ALD}\left(10^{-7} \mathrm{M}\right)$ for $24 \mathrm{~h}$. At the end of the experiments, cells were stained for apoptosis. ${ }^{*} \mathrm{p}<0.05$ vs. ALD group.

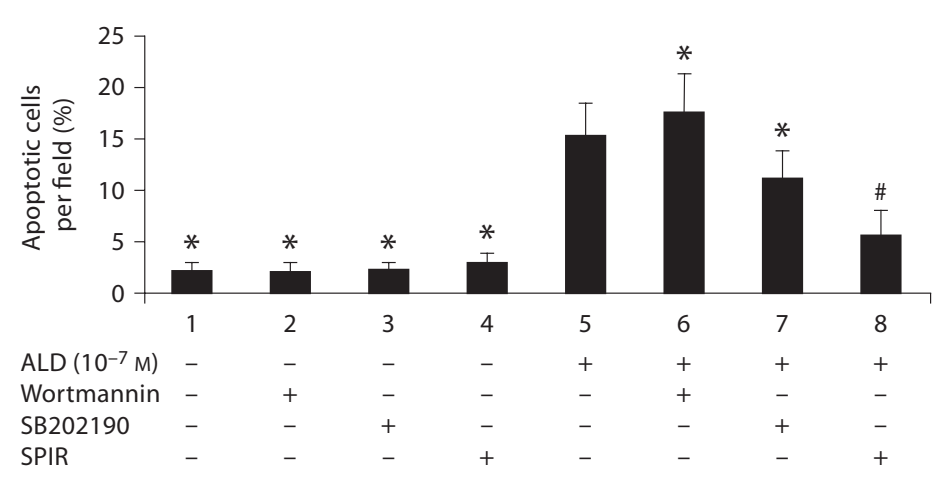

induced podocyte apoptosis, a p38MAPK inhibitor was used. Inhibiting p38MAPK by SB20 did not affect the inactivation of Akt, showing that inactivation of the Akt pathway occurs independent of the p38MAPK pathway in this process.

SPIR, which binds to cytoplasmic MR and functions as a nonspecific MR antagonist, is generally used as a diuretic to reduce the extracellular fluid volume without causing potassium depletion or hypokalemia [36]. In contrast to the well-known classic effects of SPIR, recent findings indicate that it is important for decreasing pathological tissue modifications in the cardiovascular system and the kidney [3]. The ALD receptor antagonist, SPIR, not only slows the progression of glomerulosclerosis, but also induces regression of existing glomerulosclerosis in rats [7]. SPIR can also protect cardiomyocytes and human umbilical vein endothelial cells from apoptosis $[37,38]$. In our study, we added SPIR to evaluate its influence on ALD-induced podocyte apoptosis. The results indicate that SPIR limits the apoptotic effect of ALD on podocytes. The mechanism underlying this phenomenon is not clear. Interestingly, adding SPIR reverses Akt and p38MAPK expression in podocytes incubated with ALD. Since a cross-talk exists between p38MAPK cascade and MR pathways in some kinds of cell strains [39], we hypothesize that this antiapoptotic effect of SPIR in podocytes may take place in a MR-dependent or -independent manner.

In conclusion, ALD induces podocyte apoptosis in a time- and dose-dependent manner in vitro. This effect of ALD seems to be mediated through the inactivation of PI3-K/Akt and the activation of p38MAPK independent- 
ly. ALD-induced podocyte apoptosis is partially attenuated by ALD cytoplasmic MR antagonist SPIR, positioning this compound as a promising target of intervention in renal disease.

\section{Acknowledgements}

This work was supported by the National Nature Science Foundation Grants of China (30370656 and 30871167) to Dr. Ding as well as by a grant (R01 DA 12111) from the National Institutes of Health, Bethesda, Md., USA, to Dr. Singhal.

\section{References}

1 Espiner E, Nicholls M: The Renin Angiotensin System. London, Gower Medical Publishing, 2002, pp 1-33.

2 Brown NJ: Aldosterone and end-organ damage. Curr Opin Nephrol Hypertens 2005; 14 : 235-241.

-3 Miric G, Dallemagne C, Endre Z, et al: Reversal of cardiac and renal fibrosis by pirfenidone and spironolactone in streptozotocin-diabetic rats. Br J Pharmacol 2001;133: 687-694.

-4 Rocha R, Chander PN, Zuckerman A, et al: Role of aldosterone in renal vascular injury in stroke-prone hypertensive rats. Hypertension 1999;33:232-237.

5 Sun Y, Zhang J, Lu L, et al: Aldosterone-induced inflammation in the rat heart: role of oxidative stress. Am J Pathol 2002;161:17731781.

-6 Furukawa T, Ohno S, Oguchi H, et al: Morphometric study of glomerular slit diaphragms fixed by rapid-freezing and freezesubstitution. Kidney Int 1991;40:621-624.

7 Aldigier JC, Kanjanbuch T, Ma LJ, et al: Regression of existing glomerulosclerosis by inhibition of aldosterone. J Am Soc Nephrol 2005; 16:3306-3314.

-8 Rocha R, Stier CT Jr, Kifor I, et al: Aldosterone: a mediator of myocardial necrosis and renal arteriopathy. Endocrinology 2000;141: 3871-3878.

-9 Boldyreff B, Wehling M: Non-genomic actions of aldosterone: mechanisms and consequences in kidney cells. Nephrol Dial Transplant 2003;18:1693-1695.

10 Haseroth K, Gerdes D, Berger S, et al: Rapid nongenomic effects of aldosterone in mineralocorticoid-receptor-knockout mice. Biochem Biophys Res Commun 1999;266:257261.

11 Kriz W, Gretz N, Lemley KV: Progression of glomerular diseases: is the podocyte the culprit? Kidney Int 1998;54:687-697.

-12 Schiffer M, Bitzer M, Roberts IS, et al: Apoptosis in podocytes induced by TGF-beta and Smad7. J Clin Invest 2001;108:807-816.

13 Ding G, Reddy K, Kapasi AA, et al: Angiotensin II induces apoptosis in rat glomerular epithelial cells. Am J Physiol Renal Physiol 2002;283:F173-F180.

14 Wada T, Pippin JW, Marshall CB, et al: Dexamethasone prevents podocyte apoptosis induced by puromycin aminonucleoside: role of p53 and Bcl-2-related family proteins. J Am Soc Nephrol 2005;16:2615-2625.
15 Susztak K, Raff AC, Schiffer M, et al: Glucose-induced reactive oxygen species cause apoptosis of podocytes and podocyte depletion at the onset of diabetic nephropathy. Diabetes 2006;55:225-233.

16 Burniston JG, Saini A, Tan LB, et al: Aldosterone induces myocyte apoptosis in the heart and skeletal muscles of rats in vivo. J Mol Cell Cardiol 2005;39:395-399.

17 De Angelis N, Fiordaliso F, Latini R, et al: Appraisal of the role of angiotensin II and aldosterone in ventricular myocyte apoptosis in adult normotensive rat. J Mol Cell Cardiol 2002;34:1655-1665.

18 Ding G, van Goor H, Ricardo SD, et al: Oxidized LDL stimulates the expression of TGFbeta and fibronectin in human glomerular epithelial cells. Kidney Int 1997;51:147-154.

19 Lecoeur H, Prevost MC, Gougeon ML: Oncosis is associated with exposure of phosphatidylserine residues on the outside layer of the plasma membrane: a reconsideration of the specificity of the annexin V/propidium iodide assay. Cytometry 2001;44:65-72.

20 Ding G, Franki N, Kapasi AA, et al: Tubular cell senescence and expression of TGF-beta 1 and $\mathrm{p} 21$ (WAF1/CIP1) in tubulointerstitial fibrosis of aging rats. Exp Mol Pathol 2001;70: $43-53$.

21 Furukawa T, Ohno S, Oguchi H, et al: Morphometric study of glomerular slit diaphragms fixed by rapid-freezing and freezesubstitution. Kidney Int 1991;40:621-624.

22 Griendling KK, Harrison DG: Dual role of reactive oxygen species in vascular growth. Circ Res 1999;85:562-563.

23 Lodha S, Dani D, Mehta R, et al: Angiotensin II-induced mesangial cell apoptosis: role of oxidative stress. Mol Med 2002;8:830-840.

24 Blasi ER, Rocha R, Rudolph AE, et al: Aldosterone/salt induces renal inflammation and fibrosis in hypertensive rats. Kidney Int 2003;63:1791-1800.

25 Nagase M, Shibata S, Yoshida S, et al: Podocyte injury underlies the glomerulopathy of Dahl salt-hypertensive rats and is reversed by aldosterone blocker. Hypertension 2006; 47:1084-1093.

26 Nagase M, Fujita T: Aldosterone and glomerular podocyte injury. Clin Exp Nephrol 2008;12:233-242.

27 Lawlor MA, Alessi DR: PKB/Akt: a key mediator of cell proliferation, survival and insulin responses. J Cell Sci 2001;114:29032910.
28 Widmann C, Gibson S, Jarpe MB, et al: Mitogen-activated protein kinase: conservation of a three-kinase module from yeast to human. Physiol Rev 1999;79:143-180.

-29 Alvarado-Kristensson M, Porn-Ares MI, Grethe S, et al: p38 Mitogen-activated protein kinase and phosphatidylinositol 3-kinase activities have opposite effects on human neutrophil apoptosis. FASEB J 2002;16: 129-131.

30 Foster RR, Saleem MA, Mathieson PW, et al: Vascular endothelial growth factor and nephrin interact and reduce apoptosis in human podocytes. Am J Physiol Renal Physiol 2005;288:F48-F57.

-31 Saldeen J, Lee JC, Welsh N: Role of p38 mitogen-activated protein kinase (p38 MAPK) in cytokine-induced rat islet cell apoptosis. Biochem Pharmacol 2001;61:1561-1569.

32 Cross DA, Alessi DR, Cohen P, et al: Inhibition of glycogen synthase kinase- 3 by insulin mediated by protein kinase B. Nature 1995; 378:785-789.

-33 Carpenter CL, Duckworth BC, Auger KR, et al: Purification and characterization of phosphoinositide 3-kinase from rat liver. J Biol Chem 1990;265:19704-19711.

34 Mano A, Tatsumi T, Shiraishi J, et al: Aldosterone directly induces myocyte apoptosis through calcineurin-dependent pathways. Circulation 2004;110:317-323.

35 Lai XX, Ding GH, Huang CX, et al: Angiotensin II-induced podocyte apoptosis: role of the MAPK subtypes. Beijing Da Xue Xue Bao 2004;36:131-134.

36 Pitt B, Zannad F, Remme WJ, et al: The effect of spironolactone on morbidity and mortality in patients with severe heart failure. Randomized Aldosterone Evaluation Study Investigators. N Engl J Med 1999;341:709-717.

37 Pitt B, Leopold JA: A role for mineralocorticoid receptor blockade in the prevention of apoptosis. J Mol Cell Cardiol 2005;39:415417.

38 Williams TA, Verhovez A, Milan A, et al: Protective effect of spironolactone on endothelial cell apoptosis. Endocrinology 2006; 147:2496-2505

39 Lee YS, Kim JA, Kim KL, et al: Aldosterone upregulates connective tissue growth factor gene expression via 38 MAPK pathway and mineralocorticoid receptor in ventricular myocytes. J Korean Med Sci 2004;19:805811. 